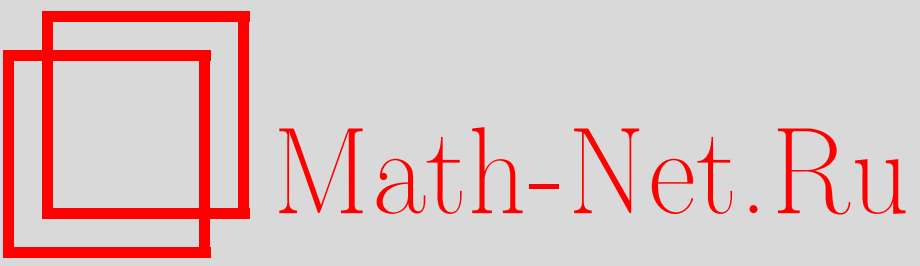

А. А. Андреев, Ю. О. Яковлева, Задача Гурса для одной системы гиперболических дифференциальных уравнений третьего порядка с двумя независимыми переменными, Вестн. Сам. гос. техн. ун-та. Сер. Физ.-мат. науки, 2011, выпуск 3()$, 35-41$

DOI: https://doi.org/10.14498/vsgtu996

Использование Общероссийского математического портала Math-Net.Ru подразумевает, что вы прочитали и согласны с пользовательским соглашением http: //www . mathnet.ru/rus/agreement

Параметры загрузки:

IP: 54.172 .240 .79

26 апреля 2023 г., 14:45:24 
УДК 517.956.3

\section{ЗАДАЧА ГУРСА ДЛЯ ОДНОЙ СИСТЕМЫ ГИПЕРБОЛИЧЕСКИХ ДИФФЕРЕНЦИАЛЬНЫХ УРАВНЕНИЙ ТРЕТЬЕГО ПОРЯДКА С ДВУМЯ НЕЗАВИСИМЫМИ ПЕРЕМЕННЫМИ}

\section{А.А. Андреев, Ю.О. Яковлева}

Самарский государственный технический университет, 443100, Самара, ул. Молодогвардейская, 244.

E-mail: julia.yakovleva@mail.ru

На основе метода Римана получено решение задачи Гурса для системы дифференииальных уравнений третъего порядка. Получена матрица Римана, выраженная через гипергеометрические функции матричного аргумента, с помощъю которой найдено решение задачи Гурса для системы линейных гиперболических уравнений третъего порядка.

Ключевые слова: система гиперболических дифференииалъных уравнений третьего порядка, сопряжённое уравнение, задача Гурса, метод Римана, матрица Римана.

Введение. Известно [1], что основной вклад в начало современной теории гиперболических уравнений в частных производных был внесён Б. Риманом, получившим в работе [2] интегральное представление задачи Коши в форме, аналогичной представлениям решений краевых задач для эллиптических уравнений второго порядка с помощью функций Грина.

Метод, применяемый Б. Риманом, предполагал существование вспомогательной функции, теперь называемой функции Римана, обладающей известными свойствами [1]. Функция Римана играет фундаментальную роль в теории линейных уравнений гиперболического типа и с её помощью удаётся, как правило, записать решение задач Коши и Гурса в явном виде (в квадрату$\operatorname{pax})$.

Однако и до сегодняшнего дня известен небольшой класс уравнений, для которых функция Римана может быть записана в замкнутом виде.

Метод Римана решения краевых задач для уравнений гиперболического типа второго порядка с двумя независимыми переменными обобщался на другие уравнения и системы. В монографиях А. В. Бицадзе [3] и И. Н. Векуа [4] приведено обобщение метода Римана на один класс гиперболических систем второго порядка с двумя независимыми переменными и кратными характеристиками, при этом показано, что вопрос о нахождении матрицы Римана редуцируется к решению системы интегральных уравнений Вольтерра второго рода, которая всегда имеет единственное решение.

Дальнейшему развитию метода Римана для уравнения гиперболического типа с двумя независимыми переменными были посвящены работы А. П. Солдатова, М.Х Шханукова [5], О. М. Джохадзе [6], О. С. Зикирова [7], где дано определение функции Римана, которая вводится как решение некоторой специальной задачи Гурса. Такой способ обеспечивает существование функ-

Александр Анатолъевич Андреев (к.ф.-м.н., доц.), доцент, каф. прикладной математики и информатики. Юлия Олеговна Яковлева, аспирант, каф. прикладной математики и информатики. 
ции Римана, но вопрос о ее явном построении остается открытым. В работах В. И. Жегалова, А.Н. Миронова и Е.А. Уткиной $[8,9]$ предлагается способ нахождения функции Римана как решения интегрального уравнения.

Известно, что с помощью интерполяционного многочлена Лагранжа-Сильвестра [10] можно определить значение аналитической функции на множестве постоянных квадратных матриц. Если ограничиться множеством матриц, являющихся значениями некоторых аналитических функций от одной матрицы, то определение легко обобщается на случай аналитических функций многих комплексных переменных, что позволяет, в свою очередь, доопределять целый ряд специальных функций на матричные значения входящих в них параметров. Некоторые примеры приведены в работах $[11,12]$, где также дано определение матрицы Римана, которое мы приведём ниже.

1. Постановка задачи. В односвязной области $D=\{(x, y): 0<x<1$, $0<y<1\}$ независимых переменных $(x, y)$ рассмотрим систему линейных уравнений третьего порядка в частных производных, не содержащую производные порядка меньше третьего:

$$
M U \equiv U_{x x y}+\Lambda U=0,
$$

где $U(x, y)$ - искомая $n$-мерная вектор-функция, $\Lambda$ - постоянная квадратная матрица порядка $n$.

Регулярным в области $D$ решением системы уравнений (1) называется вектор-функция $U(x, y)$, имеющая в $D$ все непрерывные частные производные, входящие в уравнение (1), и удовлетворяющая ей в обычном смысле.

Для системы уравнений (1) поставим следующую граничную задачу (задачу Гурса): найти регулярное в области $D$ решение $U(x, y)$ системы уравнений (1), удовлетворяющее условиям

$$
\begin{aligned}
\left.U(x, y)\right|_{x=0}=A(y), & 0 \leqslant y \leqslant 1, \\
\left.U_{x}(x, y)\right|_{x=0}=B(y), & 0 \leqslant y \leqslant 1, \\
\left.U(x, y)\right|_{y=0}=C(x), & 0 \leqslant x \leqslant 1,
\end{aligned}
$$

где $A(y), B(y), C(x)$ - заданные вектор-функции такие, что $A(0)=C(0)$, $C^{\prime}(0)=B(0)$.

Сопряжённый оператор по Лагранжу [5] для $M u$ есть

$$
M^{*} V \equiv-V_{x x y}+V \Lambda,
$$

где $V\left(x_{0}, y_{0} ; x, y\right)$ - квадратная матрица порядка $n$.

Применяя векторный аналог тождества Грина [5]

$$
V(M U)-\left(M^{*} V\right) U \equiv \frac{\partial P}{\partial x}+\frac{\partial Q}{\partial y}
$$

где $P=V U_{x y}+V_{x y} U, Q=-V_{x} U_{x}$, на области $D_{0}=\left\{(x, y): 0<x<x_{0}\right.$, $\left.0<y<y_{0}\right\}$ придём к следующему соотношению:

$$
\iint_{D_{0}}\left(V(M U)-\left(M^{*} V\right) U\right) d x d y=\int_{\Gamma} P d y-Q d x
$$

где $\Gamma=\overline{D_{0}} \backslash D_{0}$. 
Полагая, что $U(x, y)$ - решение системы уравнений $(1)$, а $V=V\left(x_{0}, y_{0} ; x, y\right)$ удовлетворяет матричному уравнению $M^{*} V=0$, после некоторых преобразований с криволинейными интегралами второго рода получим

$$
\begin{aligned}
\int_{0}^{x_{0}} & \left.V_{x}\left(x_{0}, y_{0} ; x, y\right) U_{x}(x, y)\right|_{y=0} d x- \\
- & \left.\int_{0}^{y_{0}}\left(V\left(x_{0}, y_{0} ; x, y\right) U_{x y}(x, y)+V_{x y}\left(x_{0}, y_{0} ; x, y\right) U(x, y)\right)\right|_{x=0} d y+ \\
+ & \left.\int_{0}^{y_{0}}\left(V\left(x_{0}, y_{0} ; x, y\right) U_{x y}(x, y)+V_{x y}\left(x_{0}, y_{0} ; x, y\right) U(x, y)\right)\right|_{x=x_{0}} d y- \\
& \quad-\left.\int_{0}^{x_{0}} V_{x}\left(x_{0}, y_{0} ; x, y\right) U_{x}(x, y)\right|_{y=y_{0}} d x=0 .
\end{aligned}
$$

2. Определение матрицы Римана. Матрицей Римана для уравнения (1) называется решение $V=V\left(x_{0}, y_{0} ; x, y\right)$ задачи

$$
M^{*} V=0
$$

при выполнении условий

$$
\begin{aligned}
\left.V\left(x_{0}, y_{0} ; x, y\right)\right|_{x=x_{0}} & =0, \\
\left.V\left(x_{0}, y_{0} ; x, y\right)\right|_{y=y_{0}} & =\left(x-x_{0}\right) E, \\
\left.V_{x}\left(x_{0}, y_{0} ; x, y\right)\right|_{x=x_{0}} & =E
\end{aligned}
$$

где $\left(x_{0}, y_{0}\right)$ - произвольная точка области $D, E$ - единичная матрица порядка $n$.

Интегрируя по частям полученное равенство (5) и учитывая условия (2) и (6), получим

$$
\begin{aligned}
& \int_{0}^{x_{0}} V_{x}\left(x_{0}, y_{0} ; x, 0\right) C^{\prime}(x) d x- \\
& -V\left(x_{0}, y_{0} ; 0, y_{0}\right) U_{x}\left(0, y_{0}\right)+V\left(x_{0}, y_{0} ; 0,0\right) U_{x}(0,0)- \\
& -\int_{0}^{y_{0}} V_{y}\left(x_{0}, y_{0} ; 0, y\right) U_{x}(0, y) d y-\int_{0}^{y_{0}} V_{x y}\left(x_{0}, y_{0} ; 0, y\right) A(y) d y+ \\
& +\int_{0}^{x_{0}} U_{x}\left(x, y_{0}\right) d x=0 \\
& V_{x}\left(x_{0}, y_{0} ; x_{0}, 0\right) C\left(x_{0}\right)-V_{x}\left(x_{0}, y_{0} ; 0,0\right) C(0)-\int_{0}^{x_{0}} V_{x x}\left(x_{0}, y_{0} ; x, 0\right) C(x) d x- \\
& -V\left(x_{0}, y_{0} ; 0, y_{0}\right) B\left(y_{0}\right)+V\left(x_{0}, y_{0} ; 0,0\right) B(0)+\int_{0}^{y_{0}} V_{y}\left(x_{0}, y_{0} ; 0, y\right) B(y) d y- \\
& -\int_{0}^{y_{0}} V_{x y}\left(x_{0}, y_{0} ; 0, y\right) A(y) d y-U\left(x_{0}, y_{0}\right)+A\left(y_{0}\right)=0 .
\end{aligned}
$$

Вектор-функция, являющаяся решением уравнения (1), имеет вид 


$$
\begin{aligned}
U\left(x_{0}, y_{0}\right)=V_{x} & \left(x_{0}, y_{0} ; 0,0\right) A(0)+\int_{0}^{y_{0}} V_{x}\left(x_{0}, y_{0} ; 0, y\right) A^{\prime}(y) d y- \\
& -\int_{0}^{y_{0}} V\left(x_{0}, y_{0} ; 0, y\right) B^{\prime}(y) d y+\int_{0}^{x_{0}} V_{x}\left(x_{0}, y_{0} ; x, 0\right) C^{\prime}(x) d x .
\end{aligned}
$$

3. Построение матрицы Римана. Введём новые переменные $t=x-x_{0}, s=$ $=y-y_{0}$. Матрицей Римана $V(t, s)$ является решение матричного уравнения

$$
V_{t t s}-V \Lambda=0,
$$

удовлетворяющее условиям

$$
V(0, s)=0, \quad V(t, 0)=t E, \quad V_{t}(0, s)=E .
$$

Решение задачи (8), (9) будем искать в виде мультипликативного преобразования $V=t W(\sigma)$, где $\sigma=t^{2} s$. Матричное уравнение (8) при этом преобразуется к виду

$$
4 \sigma^{2} W^{\prime \prime \prime}(\sigma)+14 \sigma W^{\prime \prime}+6 W^{\prime}-W \Lambda=0
$$

при $W(0)=E$.

Пусть $\delta \equiv \sigma \frac{d}{d \sigma}[13]$, тогда

$$
\begin{gathered}
\sigma^{2} \frac{d^{2}}{d \sigma^{2}} \equiv \delta^{2}-\delta, \\
\sigma^{3} \frac{d^{3}}{d \sigma^{3}} \equiv \delta^{3}-3 \delta^{2}+2 \delta .
\end{gathered}
$$

Подставляя (11) в (10), получим

$$
\begin{gathered}
\left(4\left(\delta^{3}-3 \delta^{2}+2 \delta\right)+14\left(\delta^{2}-\delta\right)+6 \delta\right) W=\sigma W \Lambda \\
\left(4 \delta^{2}\left(\delta+\frac{1}{2}\right) W-\sigma W \Lambda\right)=0
\end{gathered}
$$

Ищем решение матричного уравнения (12) в виде $W(\sigma)=\sum_{m=0}^{\infty} A_{m} \sigma^{m}$, где $A_{m}$ - постоянные квадратные матрицы порядка $n$. Так как

$$
4 m^{2}\left(m+\frac{1}{2}\right) A_{m}=A_{m-1}, \quad A_{0}=E,
$$

получаем следующую рекуррентную формулу:

$$
A_{m}=\frac{1}{(1)_{m}\left(\frac{3}{2}\right)_{m} m ! 2^{2 m}} \Lambda^{m} .
$$

Следовательно,

$$
W(\sigma)=\sum_{m=0}^{\infty} \frac{1}{(1)_{m}\left(\frac{3}{2}\right)_{m} m !}\left(\frac{\sigma}{4}\right)^{m} \Lambda^{m} .
$$


Пользуясь определением обобщенной гипергеометрической функции $[13,14]$, получим матрицу Римана:

$$
V\left(x_{0}, y_{0} ; x, y\right)=\left(x-x_{0}\right)_{0} F_{2}\left(1 ; \frac{3}{2} ;\left(\frac{\sigma}{4}\right) \Lambda\right) .
$$

При подстановке (13) в (7) после некоторых преобразований решение задачи Гурса уравнения (1) принимает вид

$$
\begin{aligned}
U\left(x_{0}, y_{0}\right)= & \left({ }_{0} F_{2}\left(1 ; \frac{3}{2} ; \tau_{0} \Lambda\right)+\frac{4}{3} \tau_{0}{ }_{0} F_{2}\left(2 ; \frac{5}{2} ; \tau_{0} \Lambda\right)\right) A(0)+ \\
+ & \int_{0}^{y_{0}}\left({ }_{0} F_{2}\left(1 ; \frac{3}{2} ; \tau_{1} \Lambda\right)+\frac{4}{3} \tau_{10} F_{2}\left(2 ; \frac{5}{2} ; \tau_{1} \Lambda\right)\right) A^{\prime}(y) d y+ \\
& \quad+\int_{0}^{y_{0}} x_{0}\left({ }_{0} F_{2}\left(1 ; \frac{3}{2} ; \tau_{1} \Lambda\right)\right) B^{\prime}(y) d y+ \\
& +\int_{0}^{x_{0}}\left({ }_{0} F_{2}\left(1 ; \frac{3}{2} ; \tau_{2} \Lambda\right)+\frac{4}{3} \tau_{2}{ }_{0} F_{2}\left(2 ; \frac{5}{2} ; \tau_{2} \Lambda\right)\right) C^{\prime}(x) d x
\end{aligned}
$$

где $\tau_{0}=-0,25 x_{0}^{2} y_{0}, \tau_{1}=0,25 x_{0}^{2}\left(y-y_{0}\right), \tau_{2}=-0,25\left(x-x_{0}\right)^{2} y_{0}$.

$\mathrm{C}$ помощью интерполяционного многочлена Лагранжа-Сильвестра $[10,14]$ можно определить значение функции матричного аргумента на множестве квадратных матриц следующим образом:

$$
{ }_{0} F_{2}\left(1 ; \frac{3}{2} ; \tau \Lambda\right)=\sum_{k=1}^{s} \sum_{j=1}^{m_{k}} \alpha_{k j}\left(\Lambda-\lambda_{k} E\right)^{j-1} \psi^{k}(\Lambda),
$$

где $\psi^{k}(\lambda)=\frac{\psi(\lambda)}{\left(\lambda-\lambda_{k}\right)^{m k}}, \alpha_{k j}=\frac{1}{(j-1) !}\left[\frac{0 F_{2}\left(1 ; \frac{3}{2} ; \lambda\right)}{\psi^{k}(\lambda)}\right]_{\lambda=\lambda_{k}}^{(j-1)}, j=1,2, \ldots, m_{k}, k=$ $=1,2, \ldots, s$.

Непосредственной проверкой убеждаемся, что (14) удовлетворяет задаче (1), (2). Таким образом, резюмируя вышеизложенное, основные результаты можно сформулировать в виде теоремы.

Tеорема. Если $A(y), B(y), C(x) \in C^{1}(\bar{I}), I=(0,1)$, то существует единственное решение задачи Гурса (1), (2), которое имеет вид (14).

\section{БИБЛИОГРАФИЧЕСКИЙ СПИСОК}

1. Courant R., Hilbert D. Methods of mathematical physics. Vol. II: Partial differential equations. New York - London: Interscience Publishers, 1962. 830 рр.; русск. пер.: Курант Р. Уравнения с частными производными. М.: Мир, 1964. 831 с.

2. Riemann B. Ueber die Fortpflanzung ebener Luftwellen von endlicher Schwingungsweite// Abh. d. Gött. Ges. d. Wiss., 1860. Vol. 8. Pp. 43-68.

3. Бицадзе А. В. Некоторые классы уравнений в частных производных. М.: Наука, 1981. 448 c. [Bitsadze A. V. Some classes of partial differential equations. Moscow: Nauka, 1981. 448 pp.]

4. Векуа И. Н. Новые методы решения эллиптических уравнений. М.-Л.: ОГИЗ, 1948. 296 c. [Vekua I. N. New Methods for Solving Elliptic Equations. Moscow-Leningrad: OGIZ, 1948. 296 pp.] 
5. Солдатов А.П., Шхануков М.Х. Краевые задачи с общим нелокальным условием А. А. Самарского для псевдопараболических уравнений высокого порядка // ДАН CCCP, 1987. Т. 297, №3. С. 547-552; англ. пер.: Soldatov A.P., Shkhanukov M. Kh. Boundary value problems with A. A. Samarskiî's general nonlocal condition for higher-order pseudoparabolic equations // Soviet Math. Dokl., 1988. Vol. 36, no. 3. Pp. 507-511.

6. Джсхадзе О.М. Функция Римана для гиперболических уравнений и систем высокого порядка с доминированными младшими членами // Дифферени. уравн., 2003. Т. 39, №10. C. 1366-1378; англ. пер.: Dzhokhadze O. M. The Riemann function for higher-order hyperbolic equations and systems with dominated lower terms // Differ. Equ., 2003. Vol. 39, no. 10. Pp. $1440-1453$.

7. Зикиров О.С. Локальные и нелокальные краевые задачи для гиперболических уравнений третьего порядка// Соврем. мат. прилож., 2011. Т.68. С. 101-120; англ. пер.: Zikirov O.S. Local and nonlocal boundary-value problems for third-order hyperbolic equations // J. Math. Sci.. Vol. 175, no.1. Pp. 104-123.

8. Жегалов В.И., Миронов А.Н. О задачах Коши для двух уравнений в частных производных // Изв. вузов. Матем., 2002. №5. С. 23-30; англ. пер.: Zhegalov V. I., Mironov A. N. Cauchy problems for two partial differential equations // Russian Math. (Iz. VUZ), 2002. Vol. 46, no. 5. Pp. 21-28.

9. Жегалов В.И., Уткина Е. А. Об одном псевдопараболическом уравнении третьего порядка // Изв. вузов. Матем., 1999. №10. С. 73-76; англ. пер.: Zhegalov V. I., Utkina E. A. Pseudoparabolic equation of the third order // Russian Math. (Iz. VUZ), 1999. Vol.43, no. 10. Pp. 70-73.

10. Гантмахер Ф.Р. Теория матриц. М.: Наука, 1988. 549 с. [Gantmakher F. R. Theory of matrices. Moscow: Nauka. 549 pp.]

11. Андреев A. A. Построение элементарных решений и решение задачи Коши для уравнений и систем уравнений гиперболического типа: Дис. ... канд. физ.-мат. наук. Куйбышев, 1981. 100 с. [Andreev A.A. Construction of elementary solutions and solution of Cauchy problem for equations and hyperbolic systems of equations: Ph. D. Thesis (Phys. \& Math.). Kuibyshev, 1981. 100 pp.]

12. Андреев A.А. О некоторых приложениях ассоциированных гипергеометрических функций / В сб.: Дифференциальные уравнения (математическая физика): Тез. докл. участников Куйбышевского област. межвуз. науч. совещания-семинара. Куйбышев, 1984. C. 8-9. [Andreev A. A. On some applications of associated hypergeometric functions / In: Differential Equations (Mathematical Physics). Kuibyshev, 1984. Pp. 8-9].

13. Erdélyi A., Magnus W., Oberhettinger F., Tricomi F. G. Higher transcendental functions. Vol. I / ed. H. Bateman. New York - Toronto - London: McGraw-Hill Book Co, Inc., 1953. 302 pp.; русск. пер.: Бейтмен Г., Эрдейи А. Высшие трансцендентные функции. В 3-х т. Т. 1: Гипергеометрическая функция. Функция Лежандра. М.: Наука, 1973. 296 с.

14. Андреев A.A, Огородников E.H. Матричные интегро-дифференциальные операторы и их применение // Вестн. Сам. гос. техн. ун-та. Сер. Физ.-мат. науки, 1999. № 7 . C. 27-37. [Andreev A.A., Ogorodnikov E. N. Matrix integro-differential operators and their application // Vestn. Samar. Gos. Tekhn. Univ. Ser. Fiz.-Mat. Nauki, 1999. no. 7. Pp. 27-37].

15. Кошляков Н. С., Глинер Э. Б., Смирнов М. М. Дифференциальные уравнения математической физики. М.: Государств. изд-во физ.-мат. лит., 1962. 767 c. [Koshlyakov N.S., Gliner É. B., Smirnov M. M. The differential equations of mathematical physics. Moscow: Gosudarstv. Izd-vo. Fiz.-Mat. Lit., 1962. 767 pp.]

Поступила в редакцию $11 / \mathrm{V} / 2011$;

в окончательном варианте - 11/VIII/2011. 
MSC: 35L35

\section{THE GOURSAT PROBLEM FOR ONE HYPERBOLIC SYSTEM \\ OF THE THIRD ORDER DIFFERENTIAL EQUATIONS WITH TWO} INDEPENDENT VARIABLES

\section{A. A. Andreev, J. O. Yakovleva}

Samara State Technical University,

244, Molodogvardeyskaya st., Samara, 443100, Russia.

E-mail: julia.yakovleva@mail.ru

The solution of the Goursat problem for the system of the differential equations of the third order is received on the basis of Riemann method. The Riemann matrix expressed in terms of hypergeometric functions with matrix argument is received, using it the solution of Goursat problem for the system of the linear hyperbolic equations of the third order is constructed.

Key words: hyperbolic system of the third order differential equations, adjoint equation, Goursat problem, Riemann method, Riemann matrix.

Original article submitted $11 / \mathrm{V} / 2011$; revision submitted 11/VIII/2011.

Aleksander A. Andreev (Ph.D. (Phys. \& Math.)), Associate Professor, Dept. of Applied Mathematics \& Computer Science. Julia O. Yakovleva, Postgraduate Student, Dept. of Applied Mathematics \& Computer Science. 